\title{
Hounsfield units from unenhanced 18F-FDG-PET/CT are useful in evaluating supradiaphragmatic lymph nodes in children and adolescents with classical Hodgkin's lymphoma
}

\author{
Radosław Chaber ${ }^{1, A-D, F}$, Mateusz Łasecki ${ }^{2, A-D}$, Justyna Kwaśnicka ${ }^{3, B}$, Kornelia Łach ${ }^{1, C}$, \\ Zbigniew Podgajny ${ }^{4,5, B, E}$, Cyprian Olchowy ${ }^{2, B}$, Urszula Zaleska-Dorobisz ${ }^{2, E, F}$ \\ ${ }^{1}$ Department of Pediatric Oncology and Hematology, Faculty of Medicine, University of Rzeszów, Poland \\ 2 Department of General and Pediatric Radiology, Wroclaw Medical University, Poland \\ ${ }^{3}$ Department of Pediatric Bone Marrow Transplantation, Oncology and Hematology, Wroclaw Medical University, Poland \\ ${ }^{4}$ Affidea Center of Positron Emission Tomography and Computed Tomography, Wrocław, Poland \\ ${ }^{5}$ Department of Endocrinology and Isotopes Therapy, Military Institute of Medicine, Warszawa, Poland \\ A - research concept and design; B - collection and/or assembly of data; $\mathrm{C}$ - data analysis and interpretation; \\ $D$ - writing the article; $E$ - critical revision of the article; $F$ - final approval of the article
}

Address for correspondence

Radosław Chaber

E-mail: radoslaw.chaber@gmail.com

\section{Funding sources}

None declared

Conflict of interest

None declared

Received on 0ctober 28, 2016

Reviewed on December 14, 2016

Accepted on February 14, 2017

\begin{abstract}
Background. The precise identification of the primarily-affected nodal regions in Hodgkin's lymphoma $(\mathrm{HL})$ is essential in determining the stage of the disease and the intensity of chemotherapy and radiotherapy.

Objectives. The aim of this study was to use the degree of X-ray attenuation (XRA) in Hounsfield units (HU) and the lymph node-to-muscle attenuation ratio (LN/M) in computed tomography (CT) unenhanced imaging, routinely performed with 18F-fluorodeoxyglucose positron emission tomography (18F-FDG-PET), to distinguish HL-affected supradiaphragmatic lymph nodes.

Material and methods. The study included 52 patients with classical HL treated according to the EuroNet-PHL-C1 protocol. Patients received 2 chemotherapy cycles after 18F-FDG-PET/CT testing, followed by re-examination. The lymph nodes were evaluated according to the Society for Pediatric Oncology and Hematology's GPOH-HD-2002 study and Lugano criteria as not-involved (NI-LN) and involved (I-LN).

Results. A significant difference $(p<0.001)$ was found in the XRA and LN/M values between NI-LN and I-LN before treatment and after the 2 chemotherapy cycles. The optimal cut-off point for XRA (44.7 HU) and LN/M (0.79) values distinguishing I-LN from NI-LN nodes was determined by receiver operating characteristic (ROC) analysis. After 2 cycles of chemotherapy, higher XRA $(p=0.002)$ and LN/M $(p=0.001)$ values in the group with inadequate early CTx response were found.
\end{abstract}

Conclusions. The use of XRA in HU and LN/M, together with the existing standard, can improve the qualification of supradiaphragmatic lymph nodes in HL.

Key words: children, lymph nodes, Hodgkin's lymphoma, positron-emission tomography

DOI

10.17219/acem/68990

Copyright

Copyright by Author(s)

This is an article distributed under the terms of the

Creative Commons Attribution Non-Commercial License

(http://creativecommons.org/licenses/by-nc-nd/4.0/) 


\section{Introduction}

Hodgkin's lymphoma (HL) is one of the most common neoplasms in adolescents of 15-20 years of age (approx. 12-29 cases per million per year in European countries and the USA; approx. $6 \%$ of all pediatric tumor cases). ${ }^{1,2}$

In recent decades, significant progress in the treatment of children with HL has been made. About 90-95\% of patients survive many years relapse-free. ${ }^{3}$ Currently, riskadapted therapy (chemotherapy - CTx, potentially with radiotherapy - RTx), where the intensity of treatment depends on the presence of specific prognostic factors, is a standard procedure.

A health analysis of convalescents treated in childhood for HL revealed that, despite many years of remission, a large number of them suffered from numerous late effects of the administered therapy (e.g., secondary thyroid or breast cancer, leukemia, infertility, cardiomyopathy, failure of endocrine organs, or pulmonary fibrosis). These complications significantly diminish the quality of life and may even shorten it. ${ }^{4-8}$ Contemporary regimens seek to reduce the toxicity of CTx and to completely eliminate RTx in those patients who respond well to CTx. ${ }^{9}$ If RTx is necessary, it is limited to the lowest number of fields and doses. ${ }^{10}$ The precise identification of affected lymph nodes (LN) in different regions is of fundamental importance in determining the intensity of CTx and specifying fields for possible irradiation. The selection of patients for additional RTx was based on the evaluation of morphological and functional response after the first 2 cycles of CTx.

The aim of this study was to use the degree of X-ray attenuation (XRA), expressed in Hounsfield units (HU), and the lymph node-to-muscle attenuation ratio (LN/M) in computed tomography (CT) unenhanced imaging, routinely performed with $18 \mathrm{~F}$-fluorodeoxyglucose positron emission tomography (18F-FDG-PET), to distinguish HLaffected nodes. Moreover, LN XRA values were measured after the first 2 cycles of CTx to assess morphological and functional response.

\section{Material and methods}

\section{Patients}

The study included 52 of a total of 61 consecutive patients diagnosed with classical HL between 2009 and 2015. In 7 patients it was impossible to locate the primarily affected LN or unaffected nodes. In another 2 cases, unsatisfactory technical quality made it impossible to measure XRA in control 18F-FDG-PET/CT tests. Each diagnosis was confirmed by centrally verified histopathology. The clinical characteristics of the patients are shown in Table 1. The initial staging and response to CTx assessments were performed according to the EuroNet PHL C1 protocol. ${ }^{11,12}$ After 18F-FDG-PET/CT testing, all patients received
Table 1. Characteristic of patients $(n=52)$

\begin{tabular}{|l|c|}
\hline \multicolumn{1}{|c|}{ Feature } & \\
\hline Male/female & $24 / 28$ \\
\hline Age & \\
range [years] & $4-17.5$ \\
median & 14 \\
\hline HL subtypes & \\
nodular sclerosis (NS) & 47 \\
mixed cellularity (MC) & 2 \\
\hline lymphocyte depleted (LD) & 2 \\
not done & 1 \\
\hline Initial staging (Ann Arbor) & \\
I & 1 \\
II & 24 \\
III & 13 \\
IV & 14 \\
B symptoms & 28 \\
"E" feature & 14 \\
\hline Therapeutic groups & \\
TG 1 & 9 \\
TG 2 & 15 \\
TG 3 & 28 \\
\hline Early chemotherapy response & $27 / 25$ \\
adequate/inadequate & \\
\hline
\end{tabular}

HL - Hodgkin's lymphoma; TG - therapeutic group.

2 CTx cycles - vincristine (Oncovin), etoposide, prednisone, and doxorubicin (Adriamycin - OEPA) - followed by 18 F-FDG-PET/CT re-examination. In the case of an unsatisfactory functional and/or morphological response to the administered CTx, the patient was subjected to RTx after all cycles of CTx.

\section{Analysis of CT images}

Unenhanced 18F-FDG-PET/CT studies were performed using a 16-row detector GE Discovery STE16 scanner (GE Healthcare, Milwaukee, United States) with variable voltage (100-140 keV) and intensity (10-150 mAs), single collimation width (SCW) 0.625-1.25, total collimation width (TCW) 10.0-20.0, and spiral pitch factor (SPF) 1.125-0.75. We analyzed $61 \mathrm{CT}$ components performed at the time of initial diagnosis (Fig. 1 A, B) and 61 tests evaluating the early response to $C T \mathrm{x}-$ about $2-3$ weeks after the second OEPA cycle (Fig. $1 \mathrm{C}, \mathrm{D})$. All tests were performed as part of routine clinical diagnostics. Axial sections were used for the assessment and preference was given to those having the thinnest layers, which ranged from 1.25 to $3.75 \mathrm{~mm}$, depending on the study/patient.

\section{Principles for the evaluation of lymph nodes}

Lymph nodes were evaluated according to the Society for Pediatric Oncology and Hematology's GPOH-HD 2002 study and Lugano criteria independently by 2 radiologists and a nuclear medicine specialist.,13 The Surveillance, 


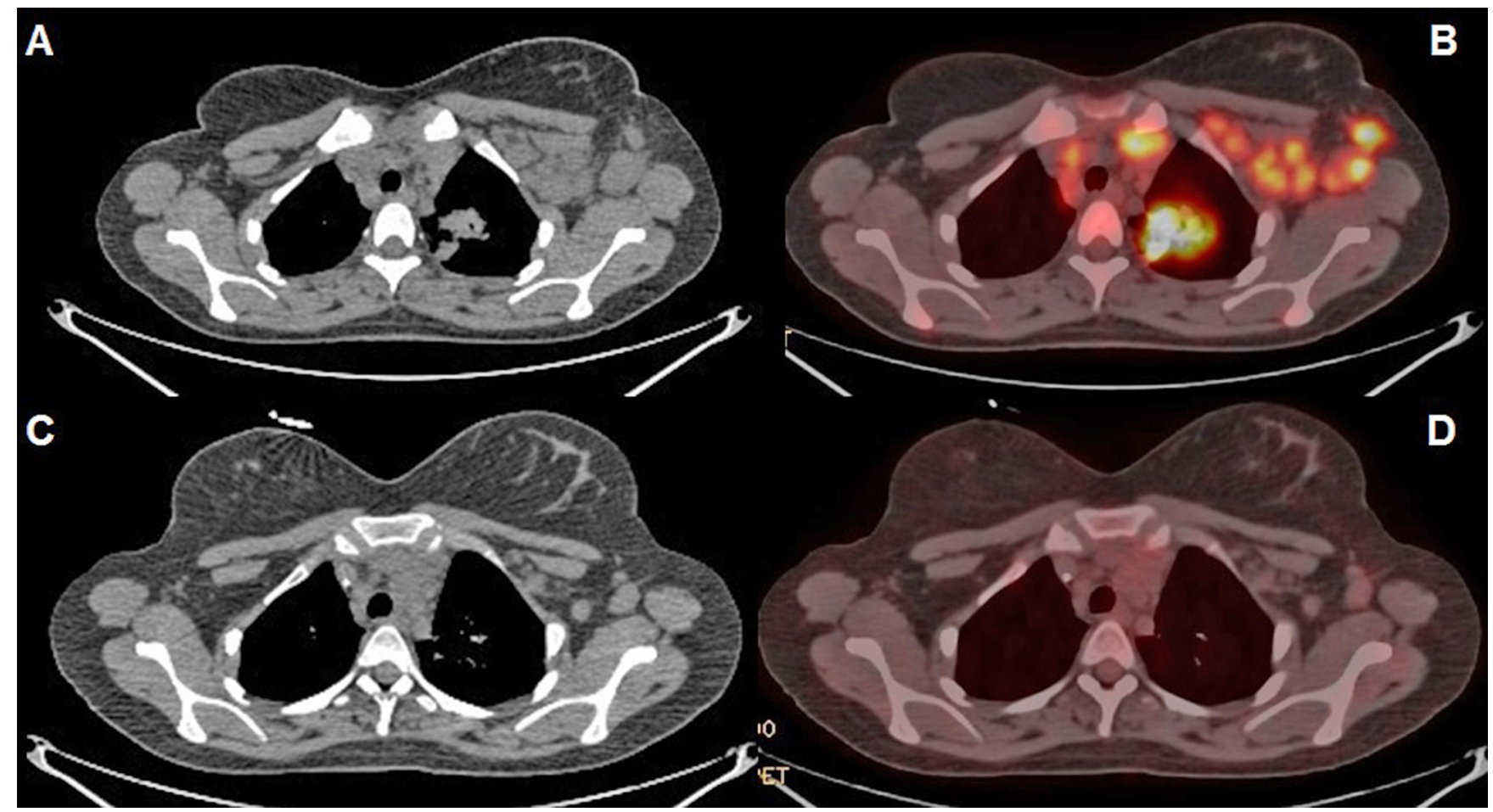

Fig. 1. A 17-year-old female with classical NS type HL; axial CT and PET-CT (fusion) images performed before (A, B) and after OEPA treatment (C, D)

A - malignant lymphadenopathy of the left axillary and subclavian lymph nodes; right axillary lymph nodes were normal; NS - nodular sclerosis; HL - Hodgkin's lymphoma, CT - computed tomography; PET - positron emission tomography; OEPA - vincristine (Oncovin), etoposide, prednisone, and doxorubicin (Adriamycin)

Epidemiology, and End Results program (SEER) database guidelines with minor modifications were adopted for the anatomic division of $\mathrm{LN}{ }^{14}$

The preferred normal LN locations were: axilliary (level 1 according to Berg), cervical (level 5 according to Som et al.), or the mandibular angle. ${ }^{15,16}$ Nodes of normal morphology and size were excluded if an affected node existed within a distance of less than $2 \mathrm{~cm}$. XRA measurement was made on an axial section of the node with the thickest cortical layer. The fatty hilum was disregarded for measurement as it would have lowered the average XRA value.

Involved nodes were selected based on the 18F-FDG-PET component, according to highest maximum standardized uptake value $\left(\mathrm{SUV}_{\max }\right)$ and the largest area of peak $\mathrm{SUV}_{\max }$ value occurrence. The most commonly elected LNs were: cervical (level 4 and 5 according to Som et al.), mediastinal (levels 2-7 according to International Symposium on Light Alloys and Composite Materials - ISLAC) and subclavian (axillary nodes level 2 and 3 according to Berg). ${ }^{15-17}$ In each case, the XRA values were assessed in the section in which the fusion image showed the highest $\mathrm{SUV}_{\max }$ value and in the 2 adjacent sections (due to the possibility of a small image shift in PET compared to the CT images, Fig. 2). ${ }^{18,19}$ The arithmetic mean was calculated using these 3 measurements. One of the factors hindering the assessment was variable upper limb positioning by the patient in individual studies.
The group of normal nodes unaffected by HL (not-involved - NI-LN) included nodes whose largest dimension was $<1 \mathrm{~cm}$ and whose SUV values did not exceed Deauville scale level 2 . Nodes $>2 \mathrm{~cm}$ rated Deauville scale level 4 or 5 were considered affected by HL (involved - I-LN). In each study, 2-5 supradiaphragmatic LNs categorized as NI-LN or I-LN were selected for analysis, and then the average XRA was calculated. In order to eliminate non-specific inter-individual variability related to different water and body fat content, the LN/M ratio was introduced. This value is obtained by dividing the average LN HU value by the $\mathrm{HU}$ value of the right erector spinae muscles. The results obtained are therefore also independent of the $\mathrm{keV}$ and mAs parameters used in the study.

The region of interest (ROI) calculation of $\mathrm{LN}$ is presented in Fig. 2.

\section{Right erector spinae muscle selection}

Measurements were made in axial sections of the CT between the places where the celiac and superior mesenteric arteries arise. This area was chosen due to the low variability of anatomical relations between the muscles and the surrounding structures in successive studies, ensuring the repeatability of measurements. The XRA measurements were performed maintaining a margin of $2-3 \mathrm{~mm}$ from fatty tissue clusters adjacent to the muscles. The region of interest included a field surface of $0.8-1 \mathrm{~cm}^{2}$ every time. 


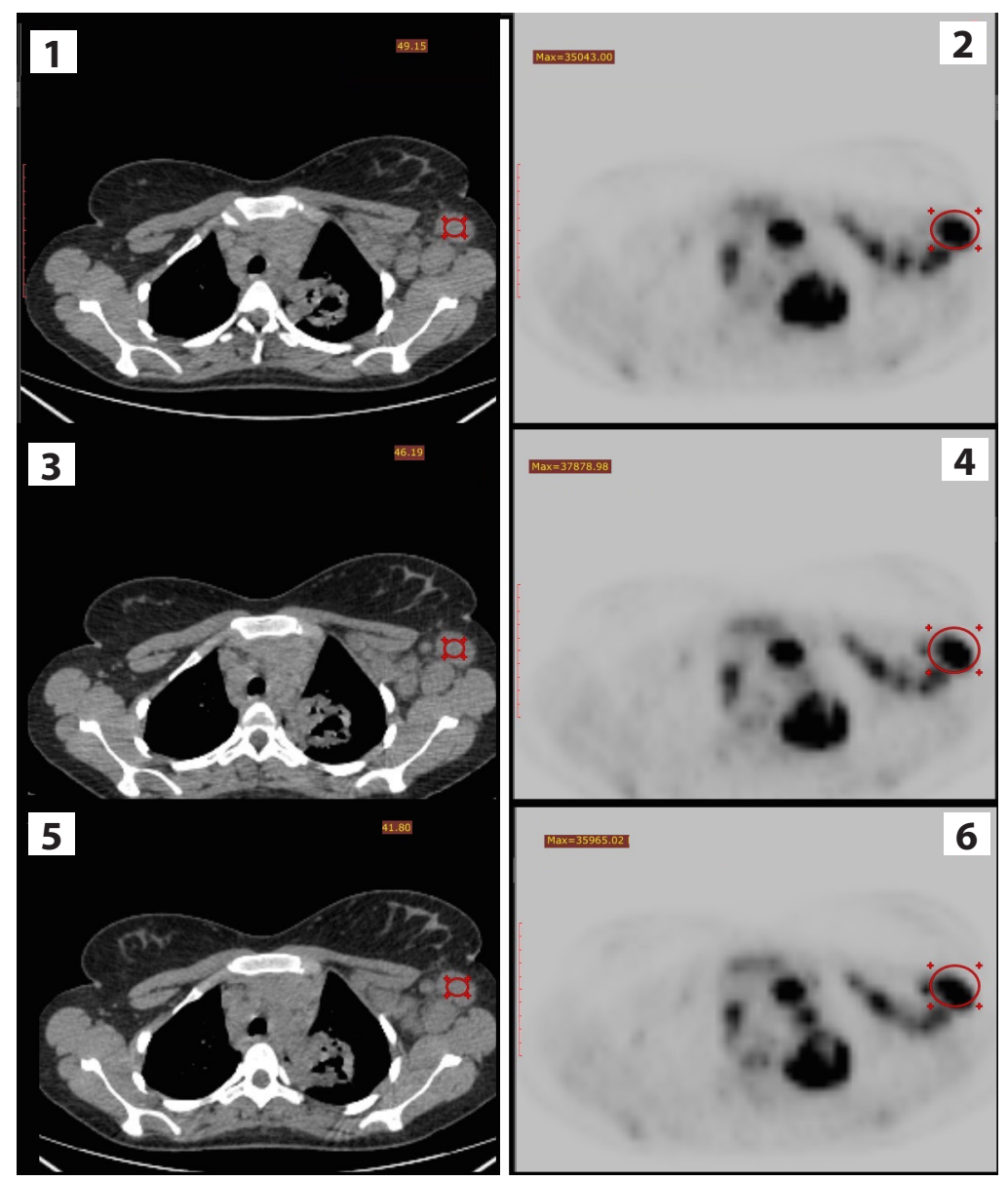

Fig. 2. This image presents the method of assessing mean density (in $\mathrm{HU}$ ) of a malignant left axillary lymph node before CTx treatment. The ROI measurement was put on the central part of the lymph node (image 3). It should cover at least half of the area of the lymph node on the axial scan with the highest SUV $\max$ value (image 4). Due to the risk of slight CT-PET image misalignment, density measurements were performed on 2 adjacent CT axial scans as well (images 1-2 and 5-6). The final $\mathrm{HU}$ value was an arithmetic mean of the 3 previous measurements

$\mathrm{HU}$ - Hounsfield units; ROI - the region of interest;

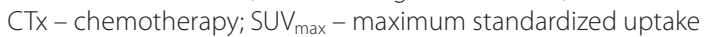
value; CT - computed tomography; PET - positron emission tomography.

Three measurements were performed from the chosen cross-section and the arithmetic mean was calculated. The presence of hardened beam artifacts or of any focal lesions (e.g., intramuscular calcification) resulted in the exclusion of measurements.

\section{Statistical analysis}

The average XRA values and the $\mathrm{LN} / \mathrm{M}$ ratios, as well as the differences depending on certain clinical parameters, were compared using Student's t-test. The Wilcoxon test for sequence pairs was used to analyze the differences of XRA and LN/M after 2 cycles OEPA relative to the primary results. The optimal cut-off point for distinguishing normal nodes from HL-affected nodes was determined using receiver operating characteristic analysis (ROC) implementing the Youden index. It was assumed that the cost of obtaining a false negative is twice as high as the cost of obtaining a false positive. The resulting area under the curve (AUC) was compared to an AUC equal to 0.5 using Hanley's algorithm. ${ }^{20}$ The level of significance was $\mathrm{p}<0.05$. The calculations were performed using STATISTICA v. 12, Medical kit v. 3.0 (StatSoft, Tulsa, USA).
Table 2. Results of NI-LN vs I-LN group $(n=52)$

\begin{tabular}{|c|c|c|c|}
\hline Variable & $\begin{array}{c}\text { Non-involved } \\
\text { lymph nodes } \\
{[\text { NI-LN] }}\end{array}$ & $\begin{array}{c}\text { Involved } \\
\text { lymph nodes } \\
{[\mathrm{I}-\mathrm{LN}]}\end{array}$ & p-value \\
\hline \multicolumn{4}{|c|}{ initial staging } \\
\hline XRA range $[\mathrm{HU}]$ & $18.5-73.7$ & $34.0-108.7$ & \multirow{2}{*}{$<0.001$} \\
\hline XRA average [HU] (SD) & $40.1(12.01)$ & $56.63(13.30)$ & \\
\hline LN/M range & $0.37-1.33$ & $0.45-2.03$ & \multirow{2}{*}{$<0.001$} \\
\hline LN/M average (SD) & $0.70(0.22)$ & $1.01(0.29)$ & \\
\hline \multicolumn{4}{|c|}{ after 2 OEPA cycles } \\
\hline XRA range $[\mathrm{HU}]$ & $5.0-56.05$ & $3.3-67.5$ & \multirow{2}{*}{$<0.001$} \\
\hline XRA average [HU] (SD) & $28.62(10.68)$ & $38.16(11.58)$ & \\
\hline LN/M range & $0.09-1.04$ & $0.06-1.22$ & \multirow{2}{*}{$<0.001$} \\
\hline LN/M average (SD) & $0.52(0.21)$ & $0.69(0.20)$ & \\
\hline \multicolumn{4}{|c|}{$\Delta=X R A(L N / M)$ after 2 OEPA cycles/XRA (LN/M) initial staging } \\
\hline range $[\mathrm{HU}]$ & $0.14-1.91$ & $0.05-1.60$ & \multirow{2}{*}{0.66} \\
\hline average [HU] (SD) & $0.75(0.30)$ & $0.71(0.28)$ & \\
\hline range $L N / M$ & $0.17-1.90$ & $0.07-1.89$ & \multirow{2}{*}{0.59} \\
\hline average LN/M (SD) & $0.76(0.34)$ & $0.73(0.31)$ & \\
\hline
\end{tabular}

XRA - X-ray attenuation; HU - Hounsfield units; LN/M - lymph node/muscle attenuation ratio; $\mathrm{NI}-\mathrm{L}$ - not-involved lymph nodes; I-LN - involved lymph nodes; SD - standard deviation; OEPA - vincristine (Oncovin), etoposide, prednisone, and doxorubicin (Adriamycin) 

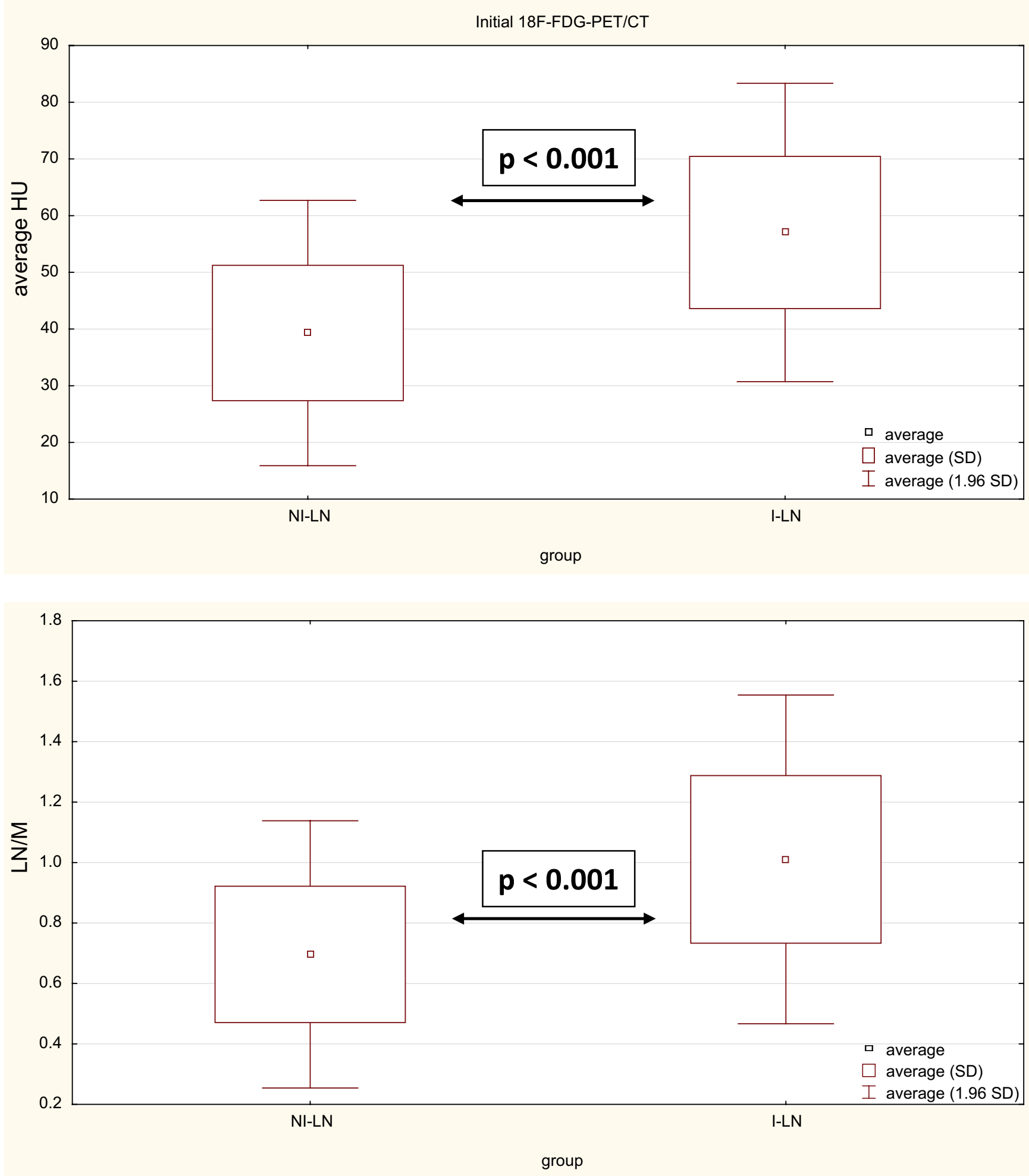

Fig. 3. Initial 18FDG-PET-CT not-involved vs involved lymph nodes [average HU; LN/M] ( $n=52)$

18FDG-PET-CT - 18F-fluorodeoxyglucose positron emission tomography; HU - Hounsfield units; LN/M - lymph node/muscle attenuation ratio; $\mathrm{NI}-\mathrm{L}$ - not-involved lymph nodes; I-LN - involved lymph nodes; SD - standard deviation.

\section{Results}

The obtained results are compared in Table 2. The average values of XRA and LN/M from I-LN and NI-LN in primary $18 \mathrm{~F}-\mathrm{FDG}-\mathrm{PET} / \mathrm{CT}$ and the changes in these parameters after 2 cycles OEPA are presented. A significant difference was found in the XRA and the average $\mathrm{LN} / \mathrm{M}$ values between the $\mathrm{LNs}$ assessed as $\mathrm{HL}$-affected due to size and metabolic activity in 18F-FDG-PET/CT vs normal LNs. This difference was significant both in studies 

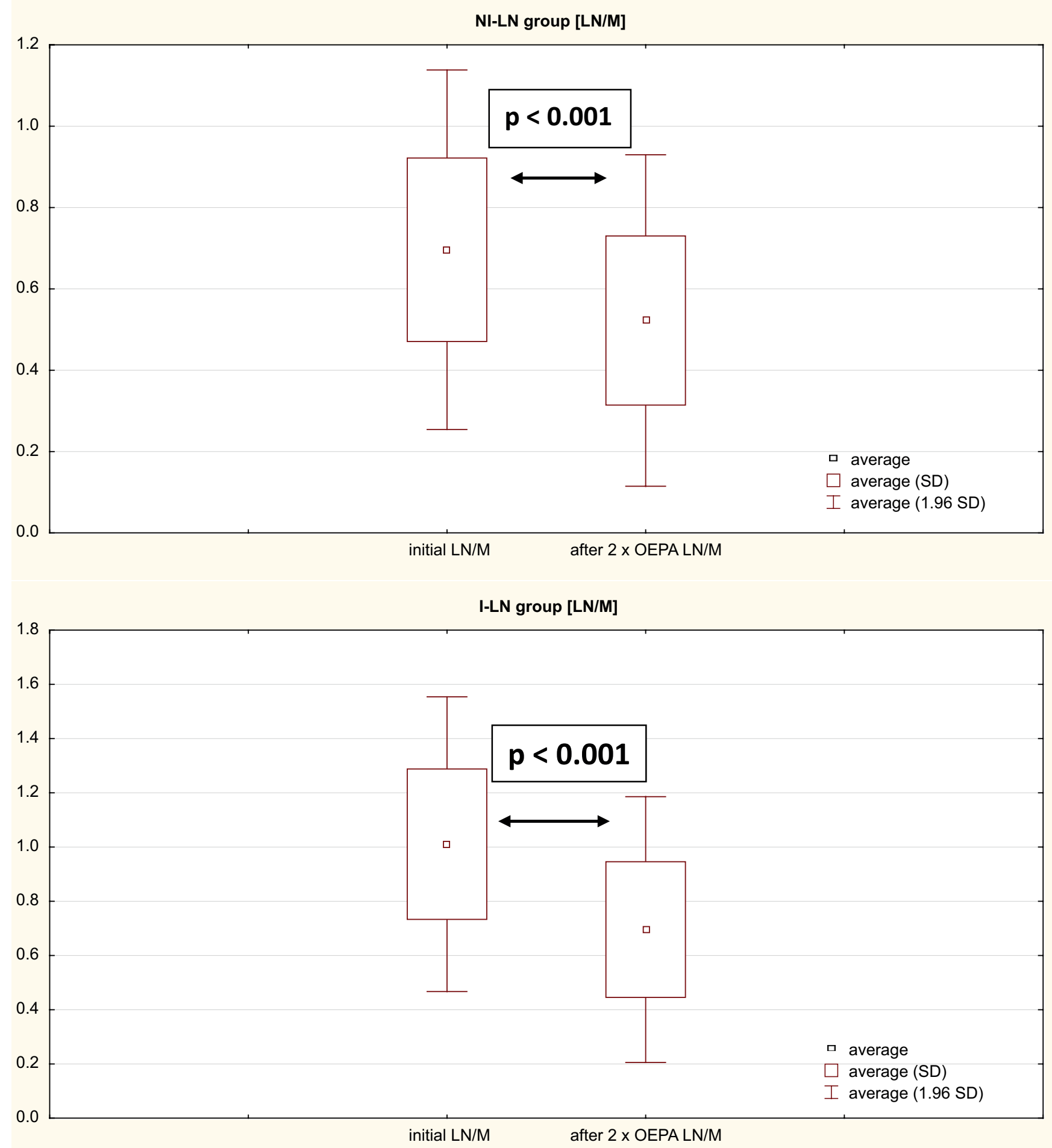

Fig. 4. 18F-FDG-PET/CT initial vs after 2 OEPA cycles [LN/M] $(n=52)$

18FDG-PET-CT - 18F-fluorodeoxyglucose positron emission tomography; OEPA - vincristine (Oncovin), etoposide, prednisone, and doxorubicin (Adriamycin) LN/M - lymph node/muscle attenuation ratio; SD - standard deviation.

performed before treatment $(\mathrm{p}<0.001$; Fig. 3$)$ and those performed during the assessment of treatment response after 2 OEPA cycles $(\mathrm{p}<0.001)$. Significantly higher values of $\mathrm{HU}$ were observed in I-LN compared to NI-LN nodes. Comparing LN/M also revealed higher values in I-LN relative to NI-LN nodes.
In the 18F-FDG-PET/CT study performed after 2 OEPA cycles, both XRA and LN/M values were significantly lower compared to baseline, in both the I-LN and the NI-LN $(\mathrm{p}<0.001$, Table 2, Fig. 4). XRA and LN/M values after $2 \mathrm{cy}-$ cles of OEPA compared with preliminary XRA and LN/M values for particular nodes $(\Delta)$ did not differ significantly 
Table 3. Initial 18F-FDG-PET/CT, I-LN group $(n=52)$

\begin{tabular}{|c|c|c|c|c|c|}
\hline \multicolumn{2}{|c|}{ Variable } & $\begin{array}{c}\mathrm{X} \text {-RA }[\mathrm{HU}] \\
\text { average } \pm \mathrm{SD}\end{array}$ & p-value & $\begin{array}{c}\mathrm{LN} / \mathrm{M} \\
\text { average } \pm \mathrm{SD}\end{array}$ & $\mathrm{p}$-value \\
\hline \multirow{2}{*}{$\begin{array}{l}\text { Age [years] } \\
\text { (number of patients) }\end{array}$} & $\leq 14(28)$ & $60.92 \pm 14.84$ & \multirow{2}{*}{0.010} & $1.12 \pm 0.32$ & \multirow{2}{*}{0.002} \\
\hline & $>14(24)$ & $51.62 \pm 9.22$ & & $0.88 \pm 0.17$ & \\
\hline \multirow{2}{*}{$\begin{array}{l}\text { Sex (number } \\
\text { of patients) }\end{array}$} & male (24) & $59.73 \pm 14.36$ & \multirow{2}{*}{0.120} & $1.04 \pm 0.29$ & \multirow{2}{*}{0.486} \\
\hline & female (28) & $53.97 \pm 11.93$ & & $0.99 \pm 0.29$ & \\
\hline \multirow{2}{*}{$\begin{array}{l}\mathrm{HL} \text { (number } \\
\text { of patients) }\end{array}$} & NS (47) & $56.18 \pm 12.82$ & \multirow{2}{*}{$0.641^{*}$} & $1.01 \pm 0.28$ & \multirow{2}{*}{$0.756^{*}$} \\
\hline & non-NS (5) & $65.48 \pm 18.04$ & & $1.07 \pm 0.38$ & \\
\hline \multirow{3}{*}{$\begin{array}{l}\text { Stage } \text { (number } \\
\text { of patients) }\end{array}$} & II (24) & $56.70 \pm 16.72$ & \multirow{3}{*}{$0.896^{\#}$} & $1.05 \pm 0.37$ & \multirow{3}{*}{$0.914^{\#}$} \\
\hline & III (13) & $56.96 \pm 11.51$ & & $0.97 \pm 0.23$ & \\
\hline & IV (14) & $56.46 \pm 8.69$ & & $0.98 \pm 0.18$ & \\
\hline \multirow{2}{*}{$\begin{array}{l}\text { Early chemotherapy } \\
\text { response (number } \\
\text { of patients) }\end{array}$} & inadequate (25) & $56.76 \pm 14.76$ & \multirow{2}{*}{0.949} & $0.99 \pm 0.28$ & \multirow{2}{*}{0.623} \\
\hline & adequate (27) & $56.52 \pm 12.07$ & & $1.03 \pm 0.30$ & \\
\hline
\end{tabular}

* the Mann-Whitney U test; " the Kruskal-Wallis test; \$ without any patient with stage I; XRA - X-ray attenuation; HU - Hounsfield units; LN/M - lymph node/muscle attenuation ratio; I-LN - involved lymph nodes; SD - standard deviation; HL - Hodgkin's lymphoma; NS - nodular sclerosis; significant results in bold.

Table 4. 18F-FDG-PET-CT after 2 OEPA cycles, I-LN group $(n=52)$

\begin{tabular}{|c|c|c|c|c|c|}
\hline \multicolumn{2}{|c|}{ Variable } & $\begin{array}{c}\mathrm{XRA}[\mathrm{HU}] \\
\text { average } \pm \mathrm{SD}\end{array}$ & $p$-value & $\begin{array}{c}\mathrm{LN} / \mathrm{M} \\
\text { average } \pm \mathrm{SD}\end{array}$ & $\mathrm{p}$-value \\
\hline \multirow{2}{*}{$\begin{array}{l}\text { Age [years] } \\
\text { (number of patients) }\end{array}$} & $\leq 14(28)$ & $37.16 \pm 12.70$ & \multirow{2}{*}{0.508} & $0.68 \pm 0.27$ & \multirow{2}{*}{0.738} \\
\hline & $>14(24)$ & $39.33 \pm 10.41$ & & $0.70 \pm 0.19$ & \\
\hline \multirow{2}{*}{$\begin{array}{l}\text { Sex (number } \\
\text { of patients) }\end{array}$} & male (24) & $36.44 \pm 9.22$ & \multirow{2}{*}{0.327} & $0.67 \pm 0.19$ & \multirow{2}{*}{0.606} \\
\hline & female (28) & $39.64 \pm 13.37$ & & $0.70 \pm 0.27$ & \\
\hline \multirow{2}{*}{$\begin{array}{l}\mathrm{HL} \text { (number } \\
\text { of patients) }\end{array}$} & NS (47) & $38.86 \pm 12.73$ & \multirow{2}{*}{$0.663^{*}$} & $0.69 \pm 0.24$ & \multirow{2}{*}{$0.686^{*}$} \\
\hline & non-NS) & $39.32 \pm 9.80$ & & $0.65 \pm 0.17$ & \\
\hline \multirow{3}{*}{$\begin{array}{l}\text { Stage } \text { (number } \\
\text { of patients) }\end{array}$} & II (24) & $38.49 \pm 10.28$ & \multirow{3}{*}{$0.335^{\#}$} & $0.69 \pm 0.23$ & \multirow{3}{*}{$0.734^{\#}$} \\
\hline & III (13) & $38.78 \pm 9.39$ & & $0.71 \pm 0.19$ & \\
\hline & IV (14) & $36.10 \pm 15.65$ & & $0.65 \pm 0.30$ & \\
\hline \multirow{2}{*}{$\begin{array}{l}\text { Early chemotherapy } \\
\text { response (number } \\
\text { of patients) }\end{array}$} & inadequate (25) & $43.26 \pm 10.35$ & \multirow{2}{*}{0.002} & $0.80 \pm 0.21$ & \multirow{2}{*}{0.001} \\
\hline & adequate (27) & $33.45 \pm 10.90$ & & $0.58 \pm 0.20$ & \\
\hline
\end{tabular}

* the Mann-Whitney U test; \# the Kruskal-Wallis test; \$ without any patient with stage I; XRA - X-ray attenuation; HU - Hounsfield units; LN/M - Iymph node/muscle attenuation ratio; I-LN - involved lymph nodes; SD - standard deviation; HL - Hodgkin's lymphoma; NS - nodular sclerosis, OEPA - vincristine (Oncovin), etoposide, prednisone, and doxorubicin (Adriamycin); significant results in bold.

in either group (I-LN vs NI-LN $\mathrm{p}=0.66$ for XRA, and $\mathrm{p}=0.59$ for the LN/M, Table 2).

In the initial 18F-FDG-PET/CT study, a significant statistical difference was found between XRA ( $p=0.001)$ and LN/M ( $\mathrm{p}=0.002)$ in the I-LN nodes in children aged up to 14 years in relation to children older than 14 years (Table 3). The difference is not as apparent after shifting the age limit to 16 years, or in the XRA for NI-LN, and in 18FDG-PET-CT after 2 OEPA cycles. There were no significant differences in $\mathrm{HU}$ values for $\mathrm{HL}$-affected nodes depending on gender, histological type, stage and responses after 2 cycles of OEPA (Table 3).

In the measurements taken after 2 cycles of OEPA, no significant difference was found in XRA depending on the analyzed clinical parameters in the I-LN group other than significantly higher XRA $(p=0.002)$ and LN/M $(p=0.001)$ values in the group with inadequate CTx response (Table 4, Fig. 5).

The optimal cut-off point for XRA and LN/M values distinguishing I-LN from NI-LN nodes was determined by ROC analysis. The greatest test accuracy was obtained by selecting baseline node XRA values of $44.7 \mathrm{HU}$ (Fig. 6, $\mathrm{AUC}=0.836,95 \%$ confidence interval $-\mathrm{CI}$ : 0.759-0.913; $\mathrm{p}<0.001)$ and $\mathrm{LN} / \mathrm{M}$ ratio of $-0.79(\mathrm{AUC}=0.804,95 \% \mathrm{CI}$ : 0.721-0.887; $\mathrm{p}<0.001)$. A comparison of the obtained ROC curve compared to a random node qualification curve demonstrated a significant statistical difference for XRA and LN/M ( $<<0.001)$.

ROC analysis was also used to assess the response of initially affected nodes to early CTx (and thus to qualify for RTx) in 18F-FDG-PET/CT after 2 OEPA cycles. The highest test accuracy was obtained when selecting a cut-off 


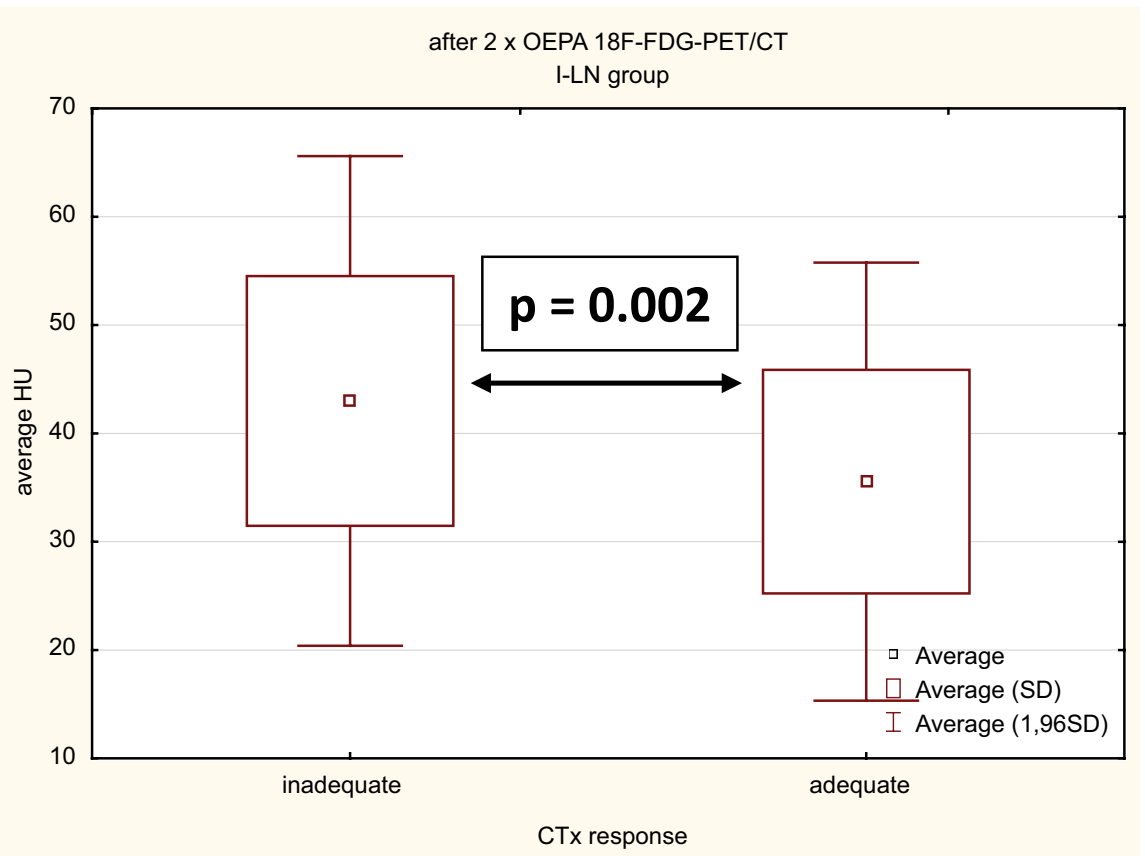

Fig. 5. 18F-FDG-PET/CT after 2 OEPA cycles, I-LN group $(n=52)$

18FDG-PET-CT - 18F-fluorodeoxyglucose positron emission tomography; OEPA vincristine (Oncovin), etoposide, prednisone, and doxorubicin (Adriamycin); I-LN - involved lymph nodes; SD - standard deviation.

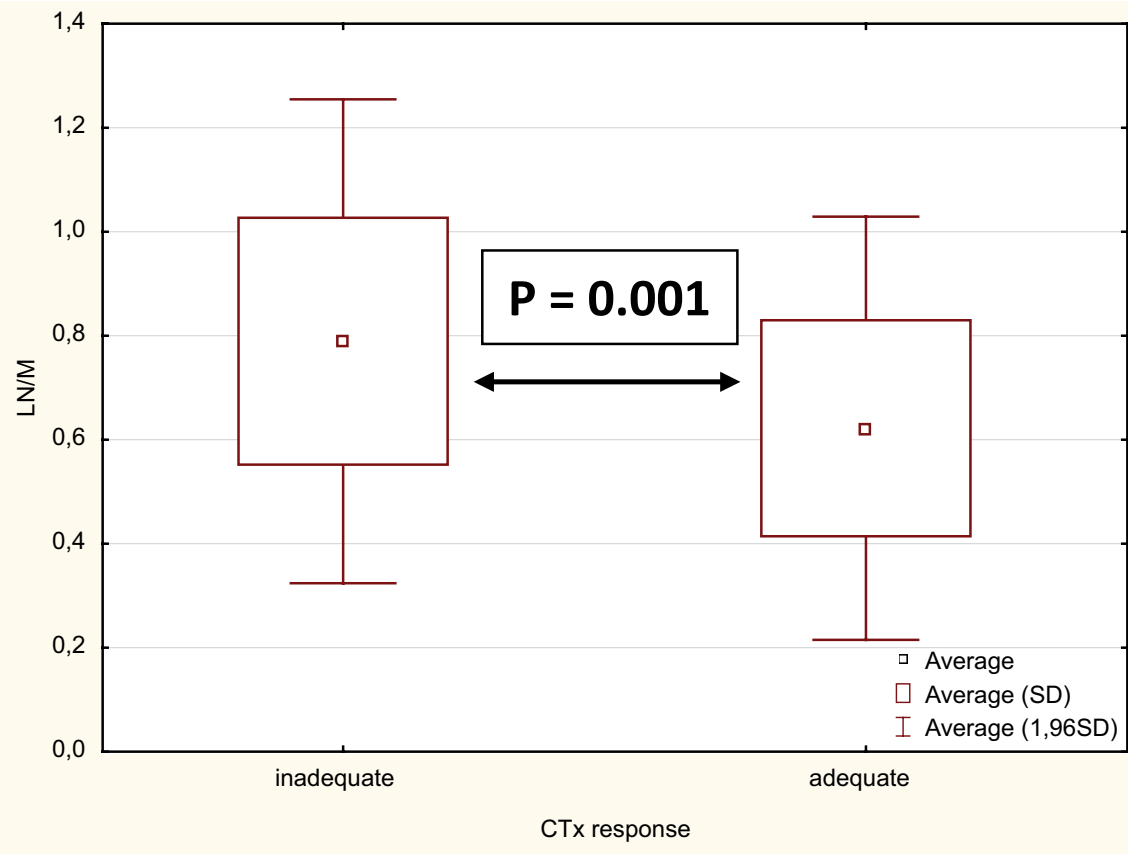

point of $34.8 \mathrm{HU}(\mathrm{AUC}=0.744,0.95 \% \mathrm{CI}: 0.608-0.879$; $\mathrm{p}=0.0004)$ and $\mathrm{LN} / \mathrm{M}$ ratio of $-0.62(\mathrm{AUC}=0.766,0.95 \%$ CI: $0.632-0.900 ; \mathrm{p}=0.0001)$. Comparing the obtained ROC curve to a random node qualification curve showed no statistically significant difference for XRA ( $p=0.12)$ or for the $\mathrm{LN} / \mathrm{M}$ ratio $(\mathrm{p}=0.11)$.

\section{Discussion}

Precise identification of all originally affected nodal regions and organs, and CTx response assessment using imaging and functional studies, are crucial in planning optimal treatment. The sensitivity and specificity of 18F-FDG-PET/CT tests in the evaluation of both initially affected LNs and the response to CTx in HL are high (90-100\% sensitivity, 80-90\% specificity) and exceed the diagnostic value of contrast CT. ${ }^{21-23}$ 18F-FDG-PET/CT interpretation can be difficult in children due to frequent respiratory infections and the associated increased metabolic activity of the lymphatic system.

We have found no publications in which an attempt has been made to use XRA in CT directly for the differentiation of affected and unaffected LNs in children and adolescents with HL. One study, investigating a population of 25 adults with lymphoma, illustrates that LN XRA values were in most cases at the same level as soft tissue - only 2 patients attained lower values. ${ }^{24}$ Similar analysis 
ROC

Youden $=0.56$

cut-off XRA: 44.70

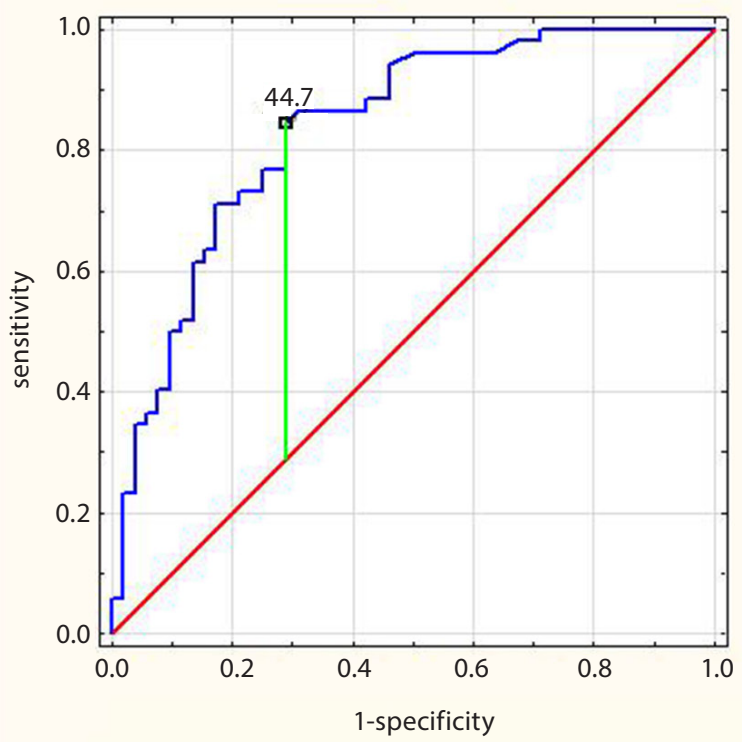

ROC

Youden $=0.50$

cut-off LN/M: 0.79

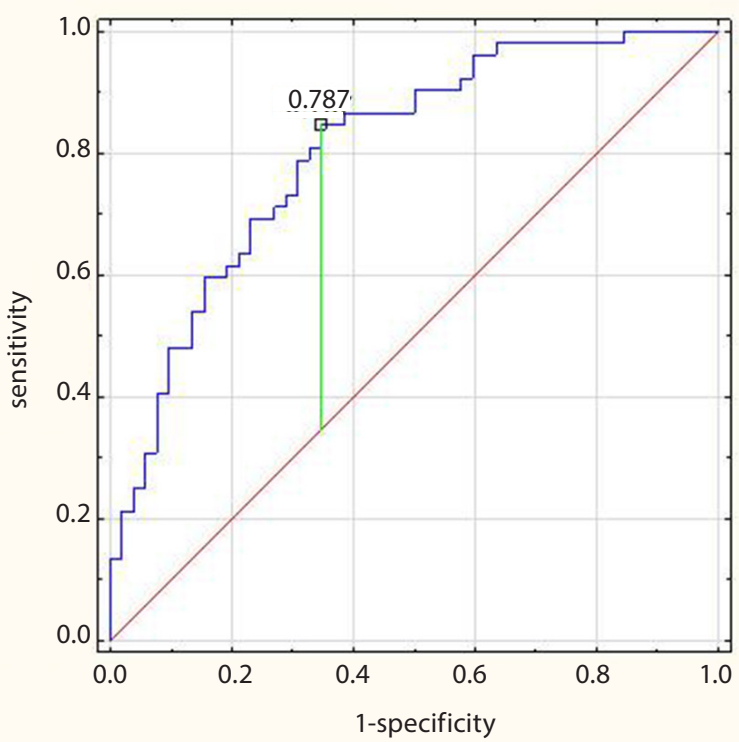

Fig. 6. Receiver operating characteristic (ROC). Initial 18F-FDG-PET/CT, NI-LN vs I-LN group ( $n=52)$

18FDG-PET-CT - 18F-fluorodeoxyglucose positron emission tomography; NI-LN - not involved lymph nodes; I-LN - involved lymph nodes.

in other neoplastic diseases also suggested this parameter's potential. In the study by Flechsig et al., which uses XRA to differentiate LNs in an orthotopic model of lung cancer in rats, it was proved that the XRA of metastatic LNs in the mediastinum was significantly higher than in those without metastatic foci. ${ }^{25}$ Yoon et al. analyzed mediastinal LNs in 674 patients with non-small-cell lung cancer. ${ }^{26}$ Nodes with XRA values $>70$ HU, despite 18FDG uptake, had a higher probability of not having metastatic foci. Measuring XRA is also used to distinguish normal from metastatic nodes in the axilliary region in patients with breast cancer. In a study by Urata et al., significantly higher XRA values in metastatic LNs as compared to unaffected nodes was shown. ${ }^{27}$ Esophageal squamous cell carcinoma is another cancer in which the evaluation of XRA can be used to differentiate between metastatic and normal LNs. In the study by Kim et al., it was shown that the XRA for metastatic nodes was significantly lower than for unaffected nodes. ${ }^{28}$

In our study, we evaluated the unenhanced component of 18F-FDG-PET/CT. One undoubted advantage is the low dosage of X-rays used, which is important due to the young age of patients and the need for periodic examinations in the monitoring of the disease. For radiological protection, it is preferable to perform magnetic resonance imaging (MRI) and 18F-FDG-PET/MRI; however, CT remains the gold standard for imaging the chest and lungs. Another advantage of using the CT component is that administering iodine contrast agents is unnecessary, unlike with classic CT. ${ }^{29}$ The administration of a contrast agent can cause many adverse effects and increases the radiation dosage in the child. ${ }^{30-32}$ The use of unenhanced CT examination eliminates the risk of tissue size and density measurement errors due to beam hardening artifacts resulting from large residual amounts of concentrated contrast in the blood vessels. ${ }^{33,34}$ An additional difficulty concerning this method is that even a slight disturbance in the contrast administration protocol or in the anatomy of the patient may significantly change the image of the scanned structures, which can cause an unwarranted increase in the planned radiation dose when administering radiation therapy. ${ }^{35,36}$

In this study we have shown that the XRA values are significantly higher in I-LN compared with NI-LN according to standard criteria. This difference is observed both in the baseline study and after 2 cycles of CTx. This may result from the different structure in nodes with active neoplastic disease compared to unaffected nodes. In neoplastic LNs, damaged internal structure with chaotic blood vessels can be seen. The presence of increased perinodular fatty tissue can also be observed. ${ }^{37}$ Nodular sclerosis Hodgkin's lymphoma classical form dominates in the pediatric population. It is characterized by a high content of fibrous connective tissue (desmoplasia), among other things. Normal LNs are characterized by a more ordered structure with a well-separated vascular hilum and regular blood flow through the node. It can be supposed that the content of lymphomatous tissue with different XRA values compared to normal tissue in the evaluated LN will determine the final XRA value. This is consistent with the observation that the XRA/(LN/M) value decreased significantly after CTx administration, both in affected and 
normal LNs, as a result of internal structural changes like desmoplasia. In patients who did not achieve an adequate morphological and functional response and were qualified for RTx, the XRA and LN/M values were higher. A weaker CTx effect on HL nodes may be visible as a "slower" decline in both the SUV and the dimensions of the I-LN, as well as the XRA (LN/M) values. The differences, however, are too small to be used as a standalone parameter for a reliable evaluation of the nodes' response to CTx.

According to our analysis, there is a visible difference in the XRA values of HL-affected nodes between children younger than 14 years and the group of older children and young adults (15-18 years old). Moving the age limit to 16 years reduces the relevance of the above difference, but it persists when the limit is shifted to 12 or 10 years. This relationship does not occur in unaffected nodes (NILN) or in imaging performed after 2 CTx cycles. This may be due to the slightly different biology and LN structure in younger children with HL.

It seems that measuring XRA/(LN/M) in 18F-FDG-PET/ $\mathrm{CT}$ without contrast, including the assessment of size and SUV, may increase the accuracy of the qualification of LNs as HL-affected or unaffected. This parameter could potentially be of use, especially in case of doubt concerning the interpretation of radiological images. Large, obvious nodal lesions of over $2 \mathrm{~cm}$ in diameter typically do not cause diagnostic dilemmas. The assessment of nodes with so-called intermediate ("unclear") dimensions $(1-2 \mathrm{~cm})$ and SUV values (2-3 points on the Deauville scale) can be more problematic. Unfortunately, a limitation of our study is the unavailability of histopathological verification for each evaluated LN. Hodgkin's lymphoma most often affects many LNs in various parts of the body. Surgical biopsy of all lesions is not clinically justified and is not routinely done. Because of the impossibility of routine histopathological confirmation, the group of intermediate LNs had to be excluded from analysis.

Another limitation is assessing the HU values in $3-\mathrm{mm}$ CT slices. Values of HU on image borders may be inaccurate for small structures such as LNs due to the partial volume effect, which specifically concerns images from PET-CT scanners. ${ }^{38,39}$ In addition, HU values depend on the CT machine, the imaging conditions, and the specifications of the image processing software, which differ in every institution. For this reason, a direct comparison of exams carried out in different centers would not be reliable. ${ }^{40}$ Introducing the LN/M and its analysis allows for the reduction of variability resulting from different technical parameters of CT scanners and exam protocols and from the impact of nonspecific external factors such as inter-individual variation.

\section{Conclusions}

Using XRA and the LN/M ratio in addition to the current standard criteria of assessing LNs in HL could be helpful and may improve the quality of their qualification as affected or unaffected. It can translate into more accurate staging and CTx response assessment in some groups of patients, and further, into reducing the incidence of the complications of RTx and CTx (the reliable determination of RTx fields and CTx intensity) and reducing the frequency of HL relapses in regions which were originally incorrectly labeled unaffected or wrongly classified as having an adequate response to CTx.

The XRA and LN/M values could be also an addition to standard criteria factors in the assessment of CTx early response in doubtful cases. Full recognition of the diagnostic value of these parameters requires further studies in larger groups of patients.

\section{References}

1. Ries LAG, Harkins D, Krapcho M, et al. (eds). SEER Cancer Statistics Review, 1975-2003, National Cancer Institute. Bethesda, MD, http:// seer.cancer.gov/csr/1975_2003/, based on November 2005 SEER data submission, posted to the SEER web site, 2006.

2. de Alarcon PA, Metzger M, Al-Rahawan MM. Pediatric Hodgkin lymphoma. Medscape News \& Perspective. http://emedicine.medscape. com/article/987101-overview\#a5. Accessed April 30, 2015.

3. National Cancer Institute. $\mathrm{PDQ}^{\circledR}$ Childhood Hodgkin Lymphoma Treatment. National Cancer Institute, Bethesda. http://www.cancer. gov/types/lymphoma/hp/child-hodgkin-treatment-pdq. Accessed February 3, 2016.

4. van Nimwegen FA, Schaapveld M, Janus CP, et al. Cardiovascular disease after Hodgkin lymphoma treatment: 40-year disease risk. JAMA Intern Med. 2015;175(6):1007-1017.

5. Chemaitilly W, Mertens AC, Mitby $P$, et al. Acute ovarian failure in the childhood cancer survivor study. J Clin Endocrinol Metab. 2006;91(5):1723-1728.

6. Constine LS, Scott Gamis A, Gross TG, et al. PDQ ${ }^{\circledR}$ Childhood Hodgkin Lymphoma Treatment. National Cancer Institute, Bethesda. http:// www.cancer.gov/types/lymphoma/hp/child-hodgkin-treatmentpdq. Accessed March 31, 2016.

7. Mauz-Körholz C, Hasenclever D, Dörffel W, et al. Procarbazine-free OEPA-COPDAC chemotherapy in boys and standard OPPA-COPP in girls have comparable effectiveness in pediatric Hodgkin's lymphoma: The GPOH-HD-2002 study. J Clin Oncol. 2010;28(23):3680-3686.

8. Maraldo MV, Brodin NP, Aznar MC, et al. Estimated risk of cardiovascular disease and secondary cancers with modern highly conformal radiotherapy for early-stage mediastinal Hodgkin lymphoma. Ann Oncol. 2013;24(8):2113-2118.

9. Raemaekers JM, Andre MP, Federico M, et al. Omitting radiotherapy in early positron emission tomography-negative stage I/II Hodgkin lymphoma is associated with an increased risk of early relapse: Clinical results of the preplanned interim analysis of the randomized EORTC/LYSA/FIL H10 trial. J Clin Oncol. 2014;32(12):1188-1194.

10. Specht $L$, Yahalom J, Illidge $T$, et al. Modern radiation therapy for Hodgkin lymphoma: Field and dose guidelines from the International Lymphoma Radiation Oncology Group (ILROG). Int J Radiat Oncol Biol Phys. 2014;89(4):854-862.

11. EuroNet-Paediatric Hodgkin's Lymphoma Group. First international Inter-Group Study for classical Hodgkin's Lymphoma in Children and Adolescents EuroNet-PHL-C1. http://www.lymphome.de/Gruppen/GPOH-HD/Protokolle/EuroNet-PHL-C1_und_R1/Synopsis.pdf. Accessed September 10, 2007.

12. EuroNet-Paediatric Hodgkin's Lymphoma Group. Recommendations for the diagnostics and treatment of children and adolescents with a classical Hodgkin's Lymphoma during the interimphase between 
the end of the EuroNet-PHL-C1 Study and the start of the EuroNetPHL-C2 Study. https://www.skion.nl/workspace/uploads/EuroNetPHL-Interim-Treatment-Guidelines-2012-12-3v0-2.pdf. Accessed January 30, 2013.

13. Barrington SF, Mikhaeel NG, Kostakoglu L, et al. Role of imaging in the staging and response assessment of lymphoma: Consensus of the International Conference on Malignant Lymphomas Imaging Working Group. J Clin Oncol. 2014;32(27):3048-3058.

14. Ries LAG, Melbert D, Krapcho M, et al. (eds). SEER Cancer Statistics Review, 1975-2005, National Cancer Institute. Bethesda, MD, https:// seer.cancer.gov/csr/1975_2005/, based on November 2007 SEER data submission, posted to the SEER web site, 2008.

15. Berg JW. The significance of axillary node levels in the study of breast carcinoma. Cancer. 1995;8:776-778.

16. Som PM, Curtin HD, Mancuso AA. Imaging-based nodal classification for evaluation of neck metastatic adenopathy. Am J Roentgenol. 2000;174(3):837-844.

17. El-Sherief AH, Lau CT, Wu CC, Drake RL, Abbott GF, Rice TW. International Association for the Study of Lung Cancer (IASLC) lymph node map: Radiologic review with CT illustration. Radiographics. 2014; 34(6):1680-1691.

18. Papathanassiou D, Becker S, Amir R, Menéroux B, Liehnet JC. Respiratory motion artefact in the liver dome on FDG PET/CT: Comparison of attenuation correction with CT and a caesium external source. Eur J Nucl Med Mol Imaging. 2005;32(12):1422-1428.

19. Pettinato $C$, Nanni C, Farsad M, et al. Artefacts of PET/CT images. Biomed Imaging Interv J. 2006;2(4):e60.

20. Hanley JA, Hajian-Tilaki HO. Sampling variability of nonparametric estimates of the areas under the receiver operating characteristic curves: An update. Acad Radiol. 1997;4:49-58.

21. Schaefer NG, Taverna C, Strobel K, Wastl C, Kurrer M, Hany TF. Hodgkin disease: Diagnostic value of FDG PET/CT after first-line therapy - is biopsy of FDG-avid lesions still needed? Radiology. 2007;244(1): 257-262.

22. Jerusalem G, Beguin Y, Fassotte MF, et al. Whole-body positron emission tomography using $18 \mathrm{~F}$-fluorodeoxyglucose compared to standard procedures for staging patients with Hodgkin's disease. Haematologica. 2001;86(3):266-273.

23. Schaefer NG, Hany TF, Taverna C, et al. Non-Hodgkin lymphoma and Hodgkin disease: Coregistered FDG PET and CT at staging and restaging - do we need contrast-enhanced CT? Radiology. 2004;232(3): 823-829.

24. Pombo F, Rodriguez E, Caruncho MV, Villalva C, Crespo C. CT attenuation values and enhancing characteristics of thoracoabdominal lymphomatous adenopathies. J Comput Assist Tomogr. 1994;18(1):59-62.

25. Flechsig $P$, Choyke $P$, Kratochwil $C$, et al. Increased $X$-ray attenuation in malignant vs. benign mediastinal nodes in an orthotopic model of lung cancer. Diagn Interv Radiol. 2015;22(1):35-39.
26. Yoon Kyung K, Kyung Soo L, Byung-Tae K, et al. Mediastinal nodal staging of nonsmall cell lung cancer using integrated 18F-FDG PET/CT in a tuberculosis-endemic country: Diagnostic efficacy in 674 patients. Cancer. 2007;109(6):1068-1077.

27. Urata M, Kijima Y, Hirata M, et al. Computed tomography Hounsfield units can predict breast cancer metastasis to axillary lymph nodes. BMC Cancer. 2014;14:730.

28. Kim SA, Lee KN, Kang EJ, Kim DW, Hong SH. Hounsfield units upon $\mathrm{PET} / \mathrm{CT}$ are useful in evaluating metastatic regional lymph nodes in patients with oesophageal squamous cell carcinoma. Br J Radiol. 2012;85(1013):606-612.

29. Follows GA, Ardeshna KM, Barrington SF, et al. Guidelines for the first line management of classical Hodgkin lymphoma. Br J Haematol. 2014;166(1):34-49.

30. Dillman JR, Strouse PJ, Ellis JH, Cohan RH, Jan SC. Incidence and severity of acute allergic-like reactions to i.v. nonionic iodinated contrast material in children. AJR Am J Roentgenol. 2007;188(6):1643-1647.

31. Zo'o M, Hoermann M, Balassy C, et al. Renal safety in pediatric imaging: Randomized, double-blind phase IV clinical trial of lobitridol 300 versus lodixanol 270 in multidetector CT. Pediatr Radiol. 2011;41(11): 1393-1400.

32. Amato E, Salamone I, Naso S, Bottari A, Gaetand M, Blandino A. Can contrast media increase organ doses in CT examinations? A clinical study. AJR Am J Roentgenol. 2013;200(6):1288-1293.

33. Birnbaum BA, Hindman N, Lee J, Babb JS. Renal cyst pseudoenhancement: Influence of multidetector $\mathrm{CT}$ reconstruction algorithm and scanner type in Phantom Model 1. Radiology. 2007;244(3):767-775.

34. Boas FE, Fleischmann D. CT artifacts: Causes and reduction techniques. Imaging Med. 2012;4(2):229-240.

35. Morrill SM, Langer ML, Lane RG, Rosen II. Tissue heterogeneity effects in treatment plan optimization. Int J Radiat Oncol Biol Phys. 1994;30(3): 699-706.

36. Burridge NA, Rowbottom CG, Bur PA. Effect of contrast-enhanced CT scans on heterogeneity corrected dose computations in the lung. J App/ Clin Med Phys. 2006;7(4):1-12.

37. Elmore SA. Histopathology of the lymph nodes. Toxicol Pathol. 2006; 34(5):425-454.

38. Tan BB, Flaherty KR, Kazerooni EA, lannettoni MD. The solitary pulmonary nodule. Chest. 2003;123:89S-96S.

39. Gould MK, Maclean CC, Kuschner WG, Rydzak CE, Owens DK. Accuracy of positron emission tomography for diagnosis of pulmonary nodules and mass lesions: A meta-analysis. JAMA. 2001;285:914-924.

40. Bui AT, Taira RK. Medical data visualization: Toward integrated clinical workstations. In: Bui AT, Taira RK, eds. Medical Imaging Informatics. New York, NY: Springer Science \& Business Media; 2010:139-193. 